Samuel Miguel Tiglao, DO, FAAP; Erica S.

Meisenheimer, MD;

Robert C. Marshall, MD, MPH, MISM, FAAFP,

FAMIA

Madigan Family Medicine Residency, Joint Base Lewis-McChord, WA

DEPUTY EDITOR

Corey Lyon, DO

University of Colorado,

Family Medicine Residency,

Denver

doi: 10.12788/jfp.0188

\title{
Automated office BP measurement: The new standard in HTN screening
}

\section{Obtain greater accuracy in blood pressure measurement with an automated office device.}

\section{PRACTICE CHANGER}

Measure patients' blood pressure (BP) using an oscillometric, fully automated office BP device, with the patient sitting alone in a quiet exam room, to accurately diagnose hypertension and eliminate the "white-coat" effect.

STRENGTH OF RECOMMENDATION

B: Based on a systematic review and metaanalysis of randomized controlled trials and cohort studies. $^{1}$

Roerecke M, Kaczorowski J, Myers MG. Comparing automated office blood pressure readings with other methods of blood pressure measurement for identifying patients with possible hypertension: a systematic review and meta-analysis. JAMA Intern Med. 2019;179:351-362.

\section{ILLUSTRATIVE CASE}

A 45-year-old woman with no chronic medical illness presents to your office for her annual physical examination. After a medical assistant (MA) applies an automatic BP cuff to the patient's left arm, the BP reading is $155 / 92 \mathrm{~mm} \mathrm{Hg}$. The MA then rechecks the $\mathrm{BP}$, and this time it reads $160 / 98 \mathrm{~mm} \mathrm{Hg}$. The MA performs a manual $\mathrm{BP}$ reading, which is $158 / 90 \mathrm{~mm} \mathrm{Hg}$ (left arm) and 162/100 mm Hg (right arm). The patient denies any headache, visual changes, chest pain, or difficulty breathing and tells the MA that her BP is always high during a doctor visit. You are wondering if she has hypertension or if is this the white-coat effect.

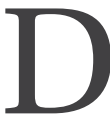

epending on the definition of hypertension, its prevalence among US adults 18 years or older varies from
$46 \%$, based on the American College of Cardiology guideline ( $\geq 130 / 80 \mathrm{~mm} \mathrm{Hg}$ ), to $29 \%$, based on the Eighth Joint National Committee (JNC-8) guideline $(\geq 140 / 90 \mathrm{~mm} \mathrm{Hg}$ for adults ages 18-59 years and $\geq 150 / 90 \mathrm{~mm} \mathrm{Hg}$ for adults $\geq 60$ years without diabetes and/or chronic kidney disease)., ${ }^{2,3}$

According to JNC-8, the prevalence is similar among men (30.2\%) and women (27.7\%) and increases with age: 18 to 39 years, $7.5 \%$; 40 to 59 years, $33.2 \%$; and $\geq 60$ years, $63.1 \% .^{3,4}$ When ranked by risk-attributable disability-adjusted life-years (DALYs), high systolic blood pressure (SBP) is the leading risk factor, accounting for 10.4 million deaths and 218 million DALYs globally in $2017 .{ }^{5}$ National medical costs associated with hypertension are estimated to account for about $\$ 131$ billion in annual health care expenditures, averaged over 12 years from 2003 to 2014 . $^{6}$

When performed correctly, the auscultatory method using a mercury sphygmomanometer correlates well with simultaneous intra-arterial BP and was considered the gold standard for office-based measurements for many years. ${ }^{7,8}$ However, significant observer-related differences in auditory acuity and terminal digit rounding are sources of inaccurate measurement. White-coat hypertension cannot be detected with this method-another significant limitation. The inaccuracy of office-based BP readings leads to concerns about hypertension being inappropriately diagnosed in patients or delays in diagnosis occurring. ${ }^{9}$ 
A proposed solution to this problem is measurement using an oscillometric sphygmomanometer. This device uses a pressure transducer to assess the oscillations of pressure in a cuff during gradual deflation; it provides accurate BP measurements when fully automated and programmed to complete several BP measurements at appropriate intervals while the patient rests alone in a quiet room. ${ }^{10}$

The accuracy of this new method was tested in a 2009 cohort study of 309 patients referred to an ambulatory blood pressure (ABP) monitoring unit at an academic hospital for diagnosis or management of hypertension. ${ }^{11}$ The study compared mean awake ABP, which continuously measures patients' BP throughout the day, manual sphygmomanometer readings taken by the patient's own physician, and an automated office blood pressure (AOBP) device called BpTRU (an automated oscillometric sphygmomanometer) while the patient rested alone in the exam room. ${ }^{11}$ The awake ABP is a federally approved standard for the diagnosis of whitecoat hypertension. ${ }^{12}$ In this study, the whitecoat response was negated with the use of the automated BpTRU device. ${ }^{11}$

A 2019 meta-analysis that included 26 studies $(\mathrm{N}=7116)$ comparing AOBP with other BP measurement techniques concluded that the use of automated oscillometric BP readings is more accurate for diagnosing hypertension and assists in negating the white-

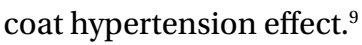

\section{STUDY SUMMARY}

Automated office BP devices are just as This systematic review and meta-analysis ( $\mathrm{N}=9279 ; 23$ cross-sectional, 1 cohort, and 7 randomized controlled trials [ $\mathrm{N}=1304]$, of which 17 studies overlapped with those included in the previously mentioned metaanalysis $^{9}$ ) compared SBP and diastolic blood pressure measured by an oscillometric AOBP device to awake or daytime ABP (continuously monitoring BP while awake, used as a standard for BP measurement), routine manual office BP, or research BP measurements.

The study also explored the protocol by which the best AOBP results could be obtained. For AOBP measurement, the included trials had no more than 2 minutes of elapsed time between individual AOBP measurements and had at least 3 AOBP readings to calculate the mean.

Compared with AOBP, in samples with an SBP of $\geq 130 \mathrm{~mm} \mathrm{Hg}$, SBP readings were significantly higher for both routine office visits (mean difference $[\mathrm{MD}]=14.5 \mathrm{~mm} \mathrm{Hg}$; 95\% CI, 11.8-17.2) and research ( $\mathrm{MD}=$ 7 mm Hg; 95\% CI, 4.9-9.1). However, no difference was found between AOBP and awake ambulatory SBP values $(\mathrm{MD}=0.3 \mathrm{~mm} \mathrm{Hg}$; $95 \% \mathrm{CI},-1.1$ to 1.7$)$. In all cases, heterogeneity of the included studies was high $\left(I^{2}\right.$ was $>75 \%$ ). There was no evidence of small-study effect or publication bias, and little evidence of potential financial bias. The most accurate methodology for AOBP measurements included multiple $\mathrm{BP}$ readings and the patient resting alone in a quiet location.

Although there was statistical heterogeneity, the results were confirmed in the authors' analysis of studies with high methodologic quality. In addition, researchers performed multiple meta-regression analyses to evaluate the statistical heterogeneity and found no significant differences based on age, body mass index, number of treated patients, gender, measurement interval, or added rest before AOBP.

\section{WHAT'S NEW}

\section{Study confirms unattended, automated office BP as preferred technique}

This is the second recent comprehensive systematic review and meta-analysis to directly compare AOBP with other common techniques of BP measurement in screening for and diagnosing hypertension in the clinical setting. ${ }^{9}$

This meta-analysis emphasized the technique (see below) by which to obtain the best AOBP vs ABP results, whereas the other meta-analysis ${ }^{9}$ did not. Thus the study provides practice-based settings with the information they need to more closely replicate the results of the studies included in the meta-analysis.

Also, the equivalency comparison with the
This metaanalysis supports the use of an automated office blood pressure device to accurately screen for hypertension and avoid the white-coat effect. 


\section{.}

Effective use of automated office blood pressure requires that one take multiple (at least 3) BP readings, 1 to 2 minutes apart, while the patient rests alone in a quiet place. more expensive and intrusive $\mathrm{ABP}$ monitoring may save money, improve patient adherence, and increase patient satisfaction. Given these advantages, along with its demonstrated accuracy, AOBP should be adopted in routine clinical practice to screen patients for hypertension.

\section{CAVEATS}

\section{Close adherence to measurement procedures is a necessity}

Effective use of AOBP in clinical practice requires close adherence to the AOBP study procedures described in this meta-analysis. These include taking multiple (at least 3) BP readings, 1 to 2 minutes apart, recorded with a fully automated oscillometric sphygmomanometer while the patient rests alone in a quiet place.

\section{CHALLENGES TO IMPLEMENTATION}

\section{Adjusting workflows, addressing cost}

Physicians may be reluctant to adopt this technique because they may not be convinced of its advantages compared with the traditional methods of recording BP and because of difficulties with implementing new rooming workflows. ${ }^{12}$ The cost of AOBP devices used in this study (Omron 907 and BpTRU; BpTRU ceased operations in 2017) were not disclosed, which may be a hindrance, as devices may cost $\$ 1000$ or more.

An online search for "automated oscillometric BP monitor" by one of the PURL authors (RCM) found oscillometric AOBP devices ranging from $\$ 150$ to $>\$ 1000$, depending on whether the device was medical grade; a search for "Omron 907" found devices for $\leq \$ 599$ on multiple sites. However, none of the lower-cost devices indicated the ability to take multiple, unattended BP readings. JFP
ACKNOWLEDGEMENT

The PURLs Surveillance System was supported in part by Grant Number UL1RR024999 from the National Center For Research Resources, a Clinical Translational Science Award to the University of Chicago. The content is solely the responsibility of the authors and does not necessarily represent the official views of the National Center For Research Resources or the National Institutes of Health. Copyright $\odot$ 2021. The Family Physicians Inquiries Network. All rights reserved.

\section{References}

1. Roerecke M, Kaczorowski J, Myers MG. Comparing automated office blood pressure readings with other methods of blood pressure measurement for identifying patients with possible hypertension: a systematic review and meta-analysis. JAMA Intern Med. 2019;179:351-362.

2. Whelton PK, Carey RM, Aronow WS, et al. 2017 ACC/AHA/AAPA/ ABC/ACPM/AGS/APhA/ASH/ASPC/NMA/PCNA guideline for the prevention, detection, evaluation, and management of high blood pressure in adults: a report of the American College of Cardiology/American Heart Association Task Force on Clinical Practice Guidelines. Hypertension. 2018;71:e13-e115. Published correction appears in Hypertension. 2018;71:e140-e144.

3. James PA, Oparil S, Carter BL, et al. 2014 evidence-based guideline for the management of high blood pressure in adults: report from the panel members appointed to the Eighth Joint National Committee (JNC 8). JAMA. 2014;311:507-520. Published correction appears in JAMA. 2014;311:1809.

4. Fryar CD, Ostchega Y, Hales CM, et al. Hypertension prevalence and control among adults: United States, 2015-2016. NCHS Data Brief. 2017;(289):1-8.

5. GBD 2017 Risk Factor Collaborators. Global, regional, and national comparative risk assessment of 84 behavioural, environmental and occupational, and metabolic risks or clusters of risks for 195 countries and territories, 1990-2017: a systematic analysis for the Global Burden of Disease Study 2017. Lancet. 2018;392:1923-1994.

6. Kirkland EB, Heincelman M, Bishu KG, et al. Trends in healthcare expenditures among US adults with hypertension: national estimates, 2003-2014. J Am Heart Assoc. 2018;7:e008731.

7. Pickering TG, Hall JE, Appel LJ, et al. Recommendations for blood pressure measurement in humans and experimental animals: part 1: blood pressure measurement in humans: a statement for professionals from the Subcommittee of Professional and Public Education of the American Heart Association Council on High Blood Pressure Research. Circulation. 2005;111:697-716.

8. Ogedegbe G, Pickering T. Principles and techniques of blood pressure measurement. Cardiol Clin. 2010;28:571-586.

9. Pappaccogli M, Di Monaco S, Perlo E, et al. Comparison of automated office blood pressure with office and out-of-office measurement techniques. Hypertension. 2019;73:481-490.

10. Reeves RA. The rational clinical examination. Does this patient have hypertension? How to measure blood pressure. JAMA. 1995;273:1211-1218.

11. Myers MG, Valdivieso M, Kiss A. Use of automated office blood pressure measurement to reduce the white coat response. $J H^{-}$ pertens. 2009;27:280-286.

12. Centers for Medicare \& Medicaid Services. Decision memo for ambulatory blood pressure monitoring (ABPM) (CAG00067R2). July 2, 2019. Accessed September 29, 2020. www.cms. gov/medicare-coverage-database/details/nca-decision-memo. aspx?NCAId $=294$ 